\title{
Control of the Oscillator Strength of the Exciton in a Single InGaN-GaN Quantum Dot
}

\author{
Anas F. Jarjour, ${ }^{1}$ Rachel A. Oliver, ${ }^{2}$ Abbes Tahraoui, ${ }^{3}$ Menno J. Kappers, ${ }^{2}$ Colin J. Humphreys, ${ }^{2}$ and Robert A. Taylor ${ }^{1, *}$ \\ ${ }^{1}$ Clarendon Laboratory, Department of Physics, University of Oxford, Parks Road, Oxford OX1 3PU, United Kingdom \\ ${ }^{2}$ Department of Materials Science and Metallurgy, University of Cambridge, Pembroke Street, Cambridge CB2 3QZ, United Kingdom \\ ${ }^{3}$ National Centre for III-V Technologies, Department of Electronic and Electrical Engineering, University of Sheffield,
}

Mappin Street, Sheffield, S1 3JD, United Kingdom

(Received 4 June 2007; published 9 November 2007)

\begin{abstract}
We report direct evidence for the control of the oscillator strength of the exciton state in a single quantum dot by the application of a vertical electric field. This is achieved through the study of the radiative lifetime of a single InGaN-GaN quantum dot in a $p-i-n$ diode structure. Our results are in good quantitative agreement with theoretical predictions from an atomistic tight-binding model. Furthermore, the increase of the overlap between the electron and hole wave functions due to the applied field is shown experimentally to increase the attractive Coulomb interaction leading to a change in the sign of the biexcitonic binding energy.
\end{abstract}

PACS numbers: 78.67.Hc, 71.70.Ej, 73.22.Dj

Nitride-based quantum dots (QDs) possess unique properties that distinguish them from the widely studied arsenide and II-VI QDs. In particular, a strong intrinsic electric field $(\sim \mathrm{MV} / \mathrm{cm})$ exists in these structures, which arises from both spontaneous polarization and piezoelectricity [1]. This field induces spatial separation between the electron and the hole wave functions, and hence leads to the appearance of a permanent electric dipole. This feature, along with the high electron and hole effective masses, would make nitride-based QDs promising candidates for quantum information processing (QIP) as they have an intrinsic built-in interaction [2]. Another advantage of the InGaN family of quantum structures is the possibility of tuning their emission across the blue and green region of the spectrum by changing the alloy content.

However, the internal electric field results in spatial separation between electrons and holes in the QD and a corresponding reduction of the overlap between their wave functions, resulting in a reduction of the oscillator strength of the interband optical transitions. The coupling of any electromagnetic field, whether it be a laser field or a vacuum field in a microcavity, is proportional to this quantity. Hence, a dynamic control of the oscillator strength is highly desirable for applications such as all-optical QIP or single-photon generation from a QD strongly coupled to a microcavity [3].

Electric fields, arising either from surface charges or from the application of an external potential, have been used in II-VI nanocrystals to manipulate electronic wave functions [4-6]. In these systems a power law decay in the luminescence is seen [7], driven by more or less strongly localized charge carriers within the nanostructures. In InGaN dots, however, the decay is a single exponential indicative of a true radiative dipole transition.

The increase in spatial separation between carriers results in a reduction of the attractive Coulomb interaction, leading to interesting phenomena such as negative biexciton binding energies $[8,9]$. Therefore, the ability to control the separation between the electrons and holes should prove very useful in the study of the Coulomb interaction of the few fermions localized in the QD.

In this Letter, we demonstrate experimentally the possibility of controlling the oscillator strength of the optical transition in a single InGaN-GaN quantum dot by applying a vertical electric field. We also show that the applied field leads to a change in the binding energy of the biexciton from negative to positive, due to an increase in the attractive Coulomb interaction between the carriers.

The sample was grown in a $p-i-n$ configuration and consisted of a QD layer in the middle of a $100 \mathrm{~nm}$ layer of intrinsic GaN, which was grown on $n$-type GaN. The structure was capped with $50 \mathrm{~nm}$-type GaN. Further details of the growth can be found in [10]. Using atomic force microscopy (AFM), the height of the QDs was found to range from 0.5 to $6.5 \mathrm{~nm}$ with mean height of $1.9 \mathrm{~nm}$ and standard deviation of $1.1 \mathrm{~nm}$. It was found that about $50 \%$ of the QDs emitted in the range $2.53-2.67 \mathrm{eV}$. When a reverse bias is applied, the external field points along the growth direction (referred to as $+\mathbf{z}$ hereafter). Microphotoluminescence (micro-PL) measurements were performed using nonresonant two-photon spectroscopy. We have found [11] that such excitation results in an almost total suppression of the background emission from the quantum well to which the QDs are coupled and yet relatively strong QD emission can still be observed. This is particularly important in the present study since the effect of the wetting layer can be readily excluded from the time-resolved measurements. Further details of the experimental arrangement can be found in [11].

Figure 1(a) shows spectra from a single QD as a function of the applied bias. It is observed that the optical transition undergoes large blueshifts (up to $\sim 60 \mathrm{meV}$ at $4 \mathrm{~V}$ ) when a reverse bias is applied. This can be explained by the partial compensation of the internal field due to the applied bias. Since a reverse bias corresponds to an applied field in the $+\mathbf{z}$ direction, this leads to the conclusion that the internal 

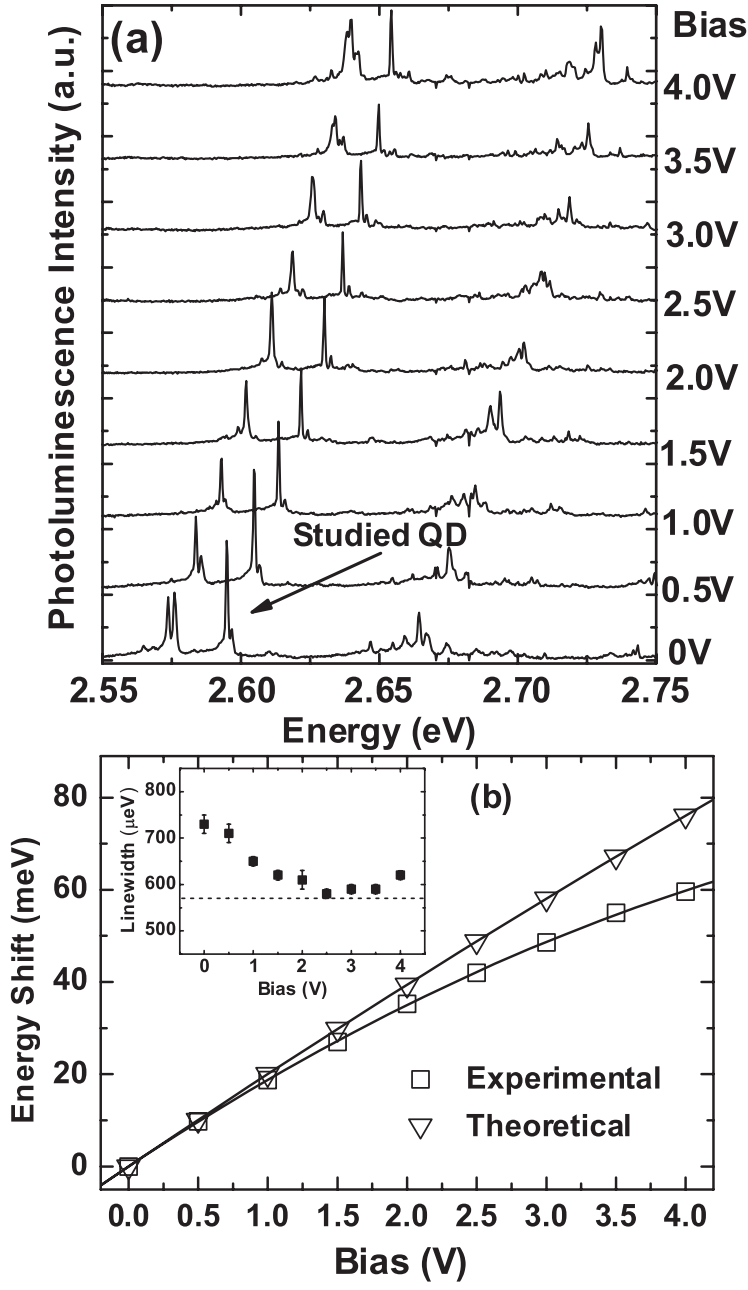

FIG. 1. (a) A series of micro-PL spectra recorded under reverse bias at $4.3 \mathrm{~K}$ under two-photon excitation. The excitation power is $38 \mathrm{~mW}$ and the excitation wavelength is $830 \mathrm{~nm}$. A large blueshift is observed. (b) The bias dependence of the energy shift of the experimental QD indicated in (a) (square symbols) and for the theoretical QD described in the text (triangular symbols). The continuous lines are a fit using a second order polynomial. The inset shows the dependence of the linewidth of the emission line from the experimental QD as obtained from a Lorentzian fit of the emission line. The dashed line represents the optical resolution of the setup, $570 \mu \mathrm{eV}$.

field is along $-\mathbf{z}$. Thus the electron should be pushed towards the top of the QD and the hole towards the bottom.

In the following, we concentrate our attention on the QD whose emission is at $2.595 \mathrm{eV}$ for an applied bias of $0 \mathrm{~V}$. The bias dependence of the energy shift of the exciton state is analyzed in Fig. 1(b). In the presence of a permanent dipole the energy shift has a linear dependence on the applied field as well as quadratic dependence, which arises from the quantum-confined Stark effect [12]. Hence the experimental data are fitted with the following equation:

$$
\Delta E(F)=\mu F+\alpha F^{2},
$$

where $\mu$ is the vertical component of the permanent dipole and $\alpha$ is the polarizability along the vertical direction. Such a dependence has been observed for both lateral $[13,14]$ and vertical $[15,16]$ applied fields for different systems of QDs. A least squares fit to the experimental data results in a value of $20.1 \pm 0.2 e \AA$ for the permanent dipole and of $-128 \pm 5 \mathrm{meV} /(\mathrm{MV} / \mathrm{cm})^{2}$ for the polarizability. An estimate of the internal piezoelectric field can be obtained by extrapolating the energy shift to its maximum value. In the case of the presented QD this value is $\sim 0.8 \mathrm{MV} / \mathrm{cm}$.

The inset of Fig. 1(b) shows the dependence of the FWHM of the emission line on the applied bias. No broadening of the emission line is observed, which is in contrast to what has been observed under applied lateral electric fields [13]. This indicates the absence of carrier tunneling processes [14] in the range of applied fields. This should be expected as the applied field would push the electron and the hole towards each other and enhance their localization in the QD. Indeed, a slight narrowing of the emission line is observed. This is also consistent with the fact that no significant change of the intensity was observed.

Time-resolved spectra of the studied QD as a function of the applied bias are presented in Fig. 2(a) and the bias dependence of the lifetime is shown in Fig. 2(b). The lifetime decreases from $13.7 \mathrm{~ns}$ to $6.3 \mathrm{~ns}$ when a bias of up to $4 \mathrm{~V}$ is applied. It can be argued that the measured lifetime is not purely radiative. However, the absence of carrier tunneling suggests that at least the nonradiative lifetime should be independent of the applied field in the considered range. Furthermore, it is shown that the measured values are in good agreement with the theoretical radiative lifetime expected. This leads to the conclusion that the nonradiative component can be excluded from the measured values. Therefore, the observed decrease in the lifetime provides direct evidence that the oscillator strength of the transition increases by a factor of 2 when half of the internal field is compensated.

In order to explain our findings, and, in particular, to check that the measured decay times correspond to the radiative lifetimes, we have employed an atomistic semiempirical $s p^{3}$ tight-binding model. Simulations of this type have been applied widely over the past few years in order to study the electronic structure of QDs [17,18]. Based on the available AFM data and our current model of the QD growth mechanism [10], we consider spherical-cap-shaped $\mathrm{In}_{x} \mathrm{Ga}_{(1-x)} \mathrm{N}$ QDs and we assume a completely random alloy. The QDs are embedded in a GaN matrix in the shape of a hexagonal prism. The details of the method are similar to those in [17]. The piezoelectric potential and the applied electric field are included by shifting the on-site orbital energy according to the equation:

$$
H_{i \gamma, i \gamma}=H_{i \gamma, i \gamma}^{0}-V^{\text {piezo }}\left(\mathbf{r}_{i}\right)+E_{z}^{\text {applied }} z_{i},
$$

where $i$ is an atomic site, $\gamma$ is an orbital type $\left(s, p_{x}, p_{y}\right.$, or $\left.p_{z}\right), H_{i \gamma, i \gamma}^{0}$ is the unstrained bulk on-site orbital energy, 

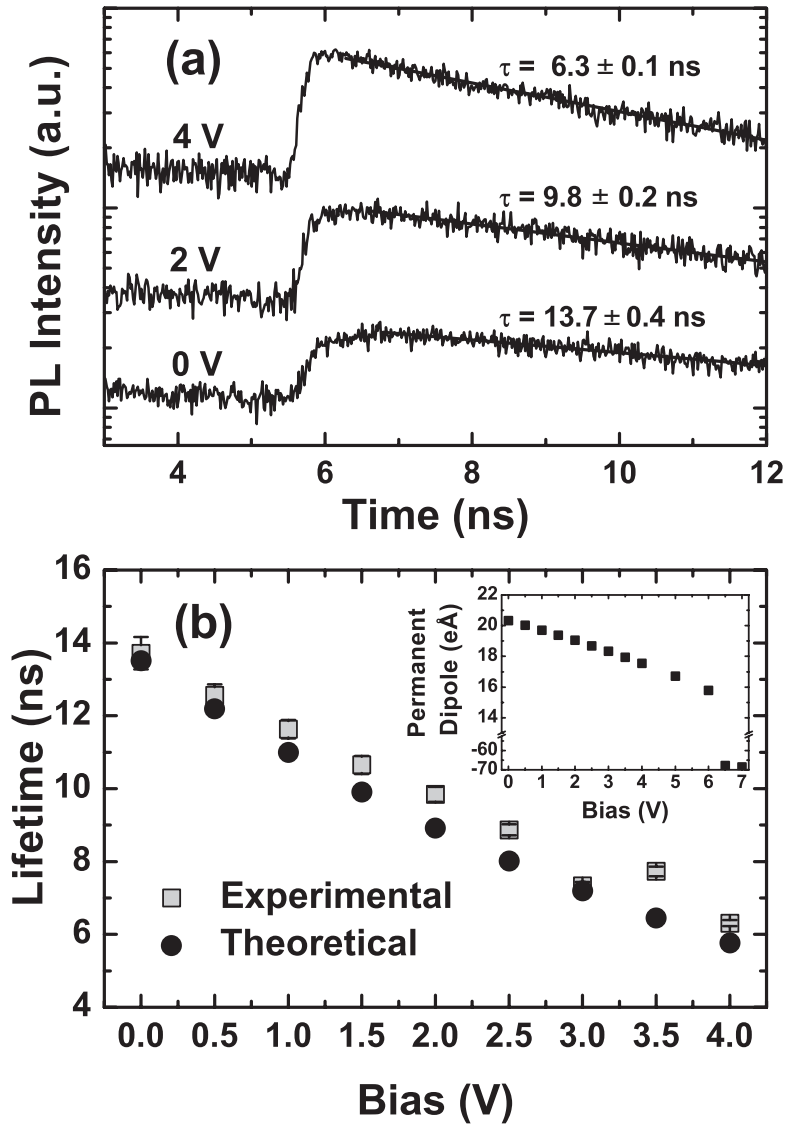

FIG. 2. (a) Time-resolved spectra (log scale) of the investigated QD for three values of the applied bias. The spectra were fitted with a single exponential. (b) The experimental (squares) and theoretical (circles) dependence of the radiative lifetime on the applied bias. The inset of (b) is the theoretical value of the $z$ component of the permanent dipole as a function of the bias.

$V^{\text {piezo }}\left(\mathbf{r}_{i}\right)$ is the piezopotential at site $i, E_{z}^{\text {applied }}$ is the applied vertical field, and $z_{i}$ is the $z$ coordinate of atom $i$.

We carried out a systematic study of the dependence of the ground state energy on the indium content and QD size. We have found that an $\operatorname{In}_{0.17} \mathrm{Ga}_{0.83} \mathrm{~N}$ QD with a base diameter of $12.5 \mathrm{~nm}$ and height of $3.1 \mathrm{~nm}$ has ground state energy of $2.607 \mathrm{eV}$ which is within $12 \mathrm{meV}$ of that in the experimental QD presented above. These values are within the size distribution inferred from AFM measurements on uncapped samples. The piezoelectric field is found to be directed along the $-\mathbf{z}$ direction, and its value at the center of the QD is $1.2 \mathrm{MV} / \mathrm{cm}$ in good agreement with the experimental estimation. The computed bias dependence of the ground state energy shift is shown in Fig. 1(b). A fit of the theoretical data using Eq. (1) gives a value for the permanent dipole of $20.4 \pm 0.24 \mathrm{e} \AA$. The same value is obtained from a direct calculation of the $z$ component of the permanent dipole from the electron and hole wave functions of the ground state at $0 \mathrm{~V}$, which confirms the consistency of the model. As for the polarizability, a value of $-34.5 \pm 0.4 \mathrm{meV} /(\mathrm{MV} / \mathrm{cm})^{2}$ is obtained from the fit, which is of the same order as the experimental value. It should be noted that the model of the electric field dependence neglects a few second order phenomena such as the effect of the applied field on the structure and on the nondiagonal elements of the Hamiltonian. The radiative lifetime of the ground state is calculated from the equation [19]:

$$
1 / \tau=\frac{n \omega^{3}|\langle 0|e \mathbf{r}| e h\rangle|^{2}}{3 \pi \epsilon_{0} \hbar c_{\mathrm{vac}}^{3}},
$$

where $\hbar \omega$ is the energy of the optical transition, $n$ is the refractive index of $\mathrm{GaN}(2.4)$, and $|\langle 0|e \mathbf{r}| e h\rangle|$ is the dipole matrix element of the transition. The calculated radiative lifetime dependence on the applied bias is shown in Fig. 2(b). Very good agreement is obtained with the experimental values. This further confirms that the measured values correspond to the radiative lifetime and hence provide a direct measurement of the increase in the oscillator strength. The increase in the wave function overlap is illustrated by the inset of Fig. 2(b), where the dependence of the calculated $z$ component of the permanent dipole on the applied field is shown. It is found that a decrease of only $15 \%$ in the separation between the electron and the hole is sufficient to increase the oscillator strength by a factor of 2 .

One interesting question that arises is whether it is in principle possible to fully compensate the internal piezoelectric field. Experimentally, we observed a rapid quenching of the QD luminescence for an applied bias above $4 \mathrm{~V}$. The theoretical simulations show that for an applied bias above $6 \mathrm{~V}$, the ground state of the hole is no longer localized in the QD as can be seen from the unphysical permanent dipole above this value in Fig. 2(b). This happens before a full compensation of the field is achieved. Hence, this imposes a fundamental limit on the extent of the control that can be applied to the oscillator strength.

As discussed above, the change of the overlap between the electron and the hole wave functions should affect the binding energy of the biexciton state. In fact, in a simple model, this binding energy is given by [20]

$$
E_{X}-E_{X X}=-\left(2 J_{e h}+J_{e e}+J_{h h}\right),
$$

where $J_{e h}$ is the electron-hole Coulomb interaction, which is negative, and $J_{e e}$ and $J_{h h}$ are the electron-electron (e-e) and hole-hole $(h-h)$ interactions, which are positive. In the absence of applied field, $\left|J_{e h}\right|$ is expected to decrease significantly due to the spatial separation between the electron and the hole, and the repulsive $e-e$ and $h$ - $h$ interactions become dominant and lead to negative binding energies [8,9]. Hence, if the applied field increases the overlap sufficiently, the attractive part of the Coulomb interaction can become dominant and a positive binding energy should be obtained. Evidence of this effect is presented below. 

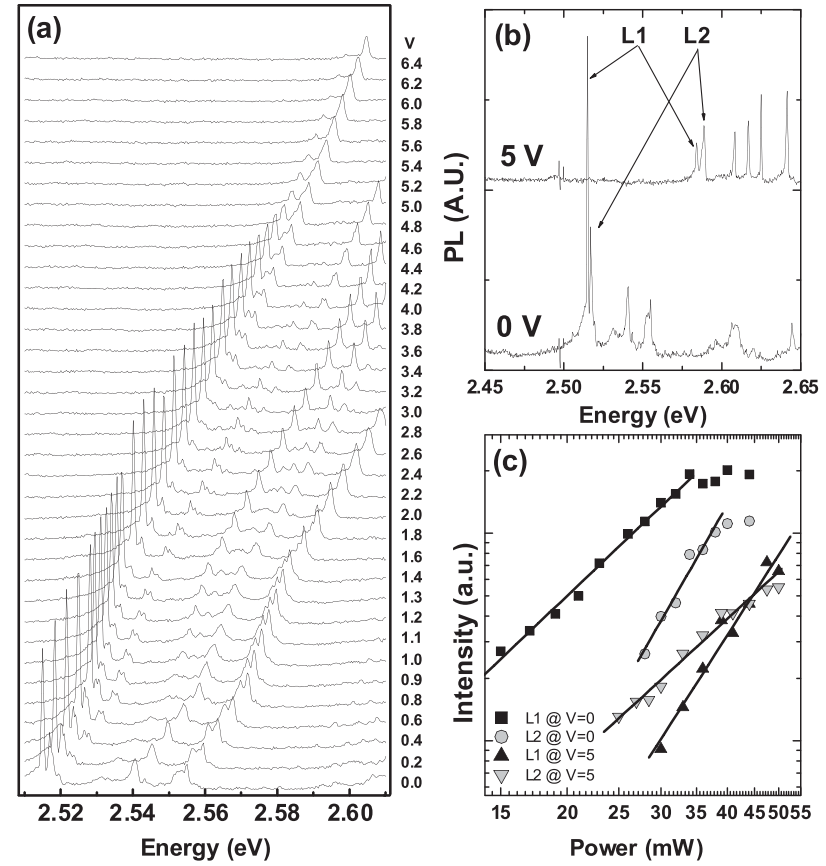

FIG. 3. (a) A series of micro-PL spectra as function of the bias from a different position recorded at $4.3 \mathrm{~K}$. In this case, the excitation power is $34 \mathrm{~mW}$ and excitation wavelength is $850 \mathrm{~nm}$. (b) An expanded portion of the spectra presented in (a) at $0 \mathrm{~V}$ and $5 \mathrm{~V}$. L1 refers to the low-energy emission line and L2 refers to the high-energy line. (c) The intensity dependence of L1 and L2 on excitation power (log-log scale) at $0 \mathrm{~V}$ and $5 \mathrm{~V}$.

Figure 3(a) shows a series of micro-PL spectra from a different position on the sample. When no field is applied, we observe a dominant emission line at $2.515 \mathrm{eV}$, which has a satellite peak to the higher energy with separation of $1.9 \mathrm{meV}$. This can be seen more clearly in Fig. 3(b). In the following, we always call the lower energy line L1 and the higher energy line L2. In order to elucidate the nature of each line, the intensity dependence on the excitation power is plotted on a log-log scale in Fig. 3(c). The linear fit shows that the intensity dependence of L1 has a scaling power of $2.4 \pm 0.1$ and hence the expected quadratic dependence for the exciton state under two-photon excitation, while the satellite line L2 has a scaling power of $4.4 \pm 0.4$ and hence can be attributed to the biexcitonic state. Therefore, when no field is applied, we observe a small negative binding energy of $1.9 \mathrm{meV}$. When a bias above $4.8 \mathrm{~V}$ is applied, the intensities of the two lines swap and L2 becomes dominant at higher fields, which can be seen in Fig. 3(b). This indicates that in this case L2 would correspond to the exciton state and L1 to the biexciton. The intensity dependence of L1 and L2 at $5 \mathrm{~V}$ shows that this is indeed the case. The scaling powers of L1 and L2 become $4.0 \pm 0.3$ and $2.3 \pm 0.1$, respectively. Hence the binding energy becomes positive, and at $5 \mathrm{~V}$ a value of $4.7 \mathrm{meV}$ is obtained. This provides additional evidence for the increase of the overlap between the carriers' wave functions.

It is observed that the swap between the intensities of the two lines occurs without crossing, which is unexpected according to the simple model described earlier. This leads to the conclusion that the exchange interaction may play an important role. Further experimental and theoretical investigation is needed to fully understand this effect.

In conclusion, we have demonstrated experimentally the possibility of controlling the oscillator strength of the optical transition in a single quantum dot by a vertical applied electric field. This is achieved via the partial compensation of the internal piezoelectric field and hence the increase of the overlap between the electron and hole wave functions. Good quantitative agreement is obtained with an atomistic tight-binding model. Such control may prove important for applications such as QD-based QIP where the control of the dipole-dipole interaction is highly desirable. We have also shown experimentally the effect of the reduction of the separation between the electron and the hole on the binding energy of the biexcitonic state in a single quantum dot.

This research is part of the QIP IRC (www.qipirc.org) supported by EPSRC (No. GR/S82176/01). We also acknowledge funding from the Clarendon Fund and the ORS scheme (A. F. J.), and from the Royal Society (R. A. O.).

*r.taylor1@ physics.ox.ac.uk

[1] F. Bernardini et al., Phys. Rev. B 56, R10024 (1997).

[2] S. De Rinaldis et al., Phys. Rev. B 69, 235316 (2004).

[3] J. P. Reithmaier et al., Nature (London) 432, 197 (2004).

[4] R. M. Kraus et al., Phys. Rev. Lett. 98, 017401 (2007).

[5] E. Rothenberg et al., Nano Lett. 5, 1581 (2005).

[6] J. Müller et al., Phys. Rev. B 72, 205339 (2005).

[7] J. M. Smith et al., arXiv:cond-mat/0604292v2.

[8] H. Schömig et al., Phys. Rev. Lett. 92, 106802 (2004).

[9] R. Seguin et al., Appl. Phys. Lett. 84, 4023 (2004).

[10] R. Oliver et al., J. Phys.: Conf. Ser. 61, 889 (2007).

[11] A. Jarjour et al., Physica (Amsterdam) 32E, 119 (2006).

[12] S. Empedocles and M. G. Bawendi, Science 278, 2114 (1997).

[13] J. Robinson et al., Appl. Phys. Lett. 86, 213103 (2005).

[14] J. Seufert et al., Physica (Amsterdam) 13E, 147 (2002).

[15] S. Raymond et al., Phys. Rev. B 58, R13415 (1998).

[16] T. Nakaoka et al., Physica (Amsterdam) 32E, 148 (2006).

[17] T. Saito and Y. Arakawa, Physica (Amsterdam) 15E, 169 (2002).

[18] S. Lee et al., Phys. Rev. B 63, 195318 (2001).

[19] A. Thränhardt et al., Phys. Rev. B 65, 035327 (2002).

[20] D. P. Williams et al., Physica (Amsterdam) 21E, 358 (2004). 